\title{
Computer Generated Holograms (CGH) for Testing, Alignment and Positioning of Astronomical and Space Mirrors Aspherical Surfaces
}

\author{
Aleksandr Semenov ${ }^{1, *}$, Magomed Abdulkadyrov ${ }^{1}$, Nikolay Dobrikov ${ }^{1}$, Aleksandr Ignatov ${ }^{1}$, Vladimir Patrikeev ${ }^{1}$, Vitaliy \\ Pridnya $^{1}$, Andrey Polyanchikov ${ }^{1}$, Ruslan Nasyrov ${ }^{2}$ \\ ${ }^{1}$ JSC Lytkarino Optical Glass Factory (LZOS), Lytkarino, 140080, Parkovaya 1, Moscow region, Russia \\ ${ }^{2}$ Institute of Automation and Electrometry of the Russian Academy of Science, 1 Koptyuga ave. Novosibirsk, 630090, Russia
}

\begin{abstract}
JSC LZOS successfully applies Computer Generated Holograms (CGH) for testing of on- and off-axis surfaces of large-dimensioned optical components for their alignment with respect to interferometer, for defining of aspherical surface optical vertex position with respect to the geometrical center and for optical systems alignment. All this allows producing of large-dimensioned aspherical on- and off-axis astronomical and space mirrors with high accuracy.
\end{abstract}

\section{Introduction}

JSC LZOS has been using Computer Generated Holograms (CGH) [1-3] for testing of surfaces of largedimensioned optical components for astronomical and space application for many years. They are applied in the process of testing of aspherical surface figure, testing of off-axis aspherical surfaces with fixing of off-axis parameters and mirror orientation, testing of aspherical surface vertex position with respect to the mirror geometrical center, mutual alignment of mirrors in test scheme, etc. Thus, CGHs have become an integral part of up-to-date testing of aspherical surfaces of largedimensioned optical mirrors and optical systems.

\section{Specific Features of Aspherical Surfaces Testing by means of CGH}

Let us consider aspherization of off-axis mirror of diameter $560 \mathrm{~mm}$ and asphericity $200 \mu \mathrm{m}$ with distance from the component center to the optical axis about 480 $\mathrm{mm}$. Final polishing was carried out with testing in scheme including CGH [4,5]. Fig. 1 shows testing scheme including CGH corrector with aligning focusing elements (Fig. 2) for $\mathrm{CGH}$ alignment with respect to interferometer. Focusing elements create specular points, according to which the component alignment with respect to the $\mathrm{CGH}$ corrector is performed, in the edge zone of the component, in the center and in the vertex position of the optical axis (Fig. 1).

Accurate and unambiguous mirror alignment in space allowed successful performance of operations on figuring of aspherical surface. The obtained quality of hyperboloid deviations from theoretical $\mathrm{RMS}=0.05 \lambda$ $(\lambda=632.8 \mathrm{~nm})$.
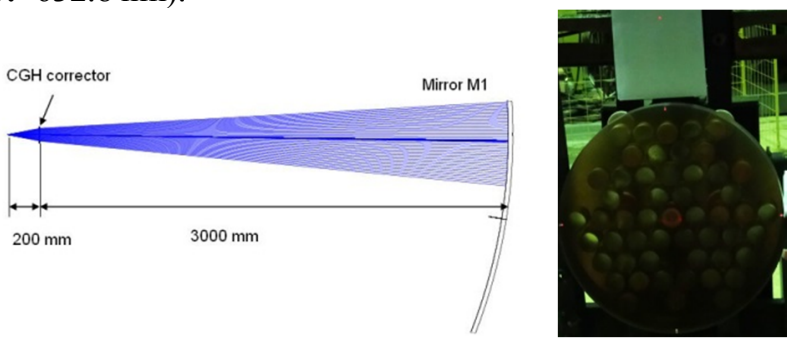

Fig 1. Testing scheme and focusing alignment elements of offaxis mirror of diameter $560 \mathrm{~mm}$
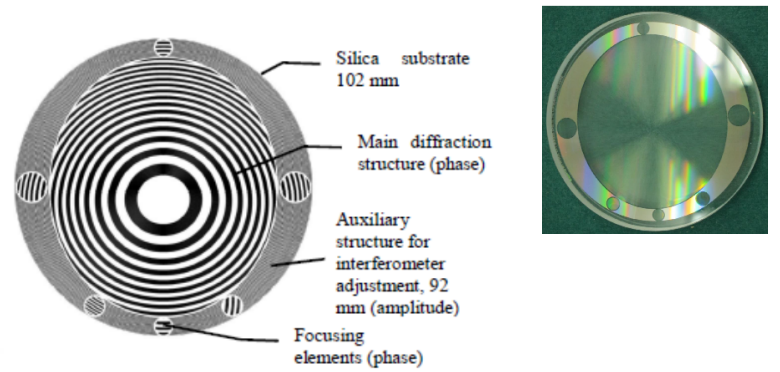

Fig. 2. CGH for off-axis mirror testing.

Another example of testing of of-axis optical elements is shown in Fig. 3. It shows testing of stereoscopic system with two off-axis mirrors, positioned symmetrically with respect to the line crossing the optical axis perpendicularly. The mirrors are installed on Whiffle tree. Then one mirror unit with support is fixed motionless, while the second one (or both) is installed on hexapod. Thus, mutual alignment of off-axis elements by means of hexapod and CGH located in the mirrors testing point is performed. $\mathrm{CGH}$ for

"Corresponding author: lastro@,1zos.ru 
mirrors testing is shown in Fig. 3. It has special auxiliary diffraction structures for the formation of crosses in the mirror area, which are used for alignment of mirrors with respect to $\mathrm{CGH}$.

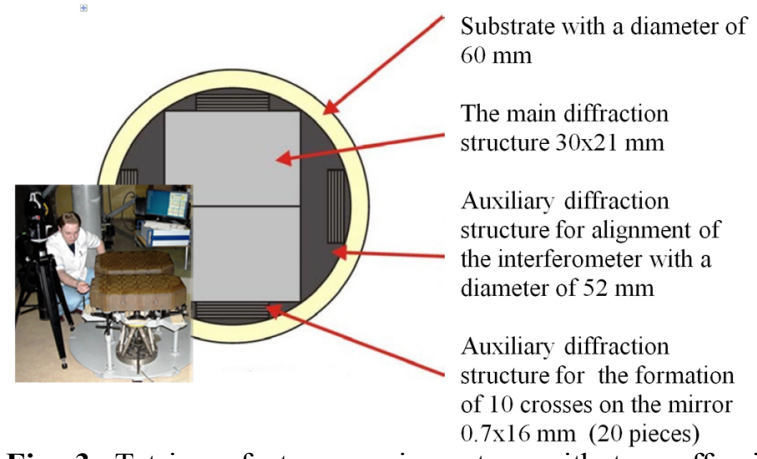

Fig. 3. Tetsing of stereoscopic system with two off-axis mirrors.

Under Contract with AMOS, JSC LZOS is producing optics for DAG Telescope (Doğu Anadolu Gözlemevi) [6]. Primary mirror DAG M1 of diameter $4000 \mathrm{~mm}$ has asphericity $139 \mu \mathrm{m}$ and aperture $\mathrm{f} / 1.8$ and very strict requirements to the accuracy of optical surface production. CGH corrector was designed and produced by the Institute of Automation and Electrometry of the Russian Academy of Science Siberian Department (Novosibirsk). It converts spherical wavefront into aspherical and back. The corrector was produced from quartz plate of diameter $102 \mathrm{~mm}$, corrector diameter is $80 \mathrm{~mm}$, the diameters of the auxiliary adjustment sphere circle are $82-92 \mathrm{~mm}$. The distance from the hologram to the focus point is $1170 \mathrm{~mm}$. In addition, this wavefront corrector has one more circular structure focusing the hologram, which creates a specular point in the mirror vertex (Fig. 4). By means of this point, we are able to define the aspherical surface vertex shift with respect to the mirror geometrical center, which is required by the specification. The accuracy of positioning of such point depends on the accuracy of $\mathrm{CGH}$ installation with respect to the interferometer. When CGH works from diverging beam, it is adjusted by means of reflecting hologram. Such reflecting hologram is calculated in such a way that an endless strip appears on it when CGH is accurately aligned with respect to the interferometer. Focusing structure for DAG M1 was made in the form of circle around the main structure. Such structure forms a point in the mirror vertex. Distance Focus-CGH $=1170$ $\mathrm{mm}$, Distance CGH-Mirror $=14274 \mathrm{~mm}$, Retro Diameter (Out-Inner) $=86-96 \mathrm{~mm}$, Focusing Diameter (Out-Inner) $=80.4-84.4 \mathrm{~mm}$.

Fig. 5 shows scheme of the focused point shift when $\mathrm{CGH}$ is tilted. It can be seen from the Figure that when CGH is tilted by angle $\alpha$, the point shift is $\mathrm{d}=\mathrm{L} * \tan (\alpha)$. Obviously, the hologram tilt when there is one strip on the reflecting structure does not depend on distance расстояния $\mathrm{F}$, it is defined by the diameter of the reflecting hologram only. The spot size is defined on the basis of Rayleigh criterion $2.44 \lambda \mathrm{L} / \mathrm{D}$, where $\mathrm{D}$ is diameter of the focusing structure and $\lambda=0.6328 \mu \mathrm{m}$ is the interferometer wavelength. In is apparent that the vertex shift $(48 \mu \mathrm{m})$ is less than the spot size $(260 \mu \mathrm{m})$. However, in practice, it is difficult to set even one strip, and there are usually three-four of them. Therefore, it is necessary to multiply the vertex shift by the number of strips. This is true for a small number of strips on interferogram.

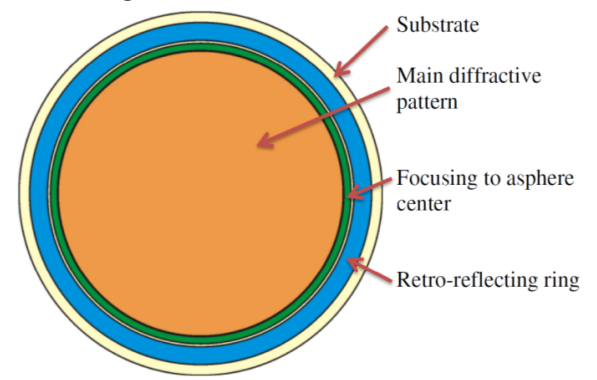

Fig. 4. Hologram layout.

Omitting a more detailed description, we can conclude that the indicated method allows defining of the mirror vertex position with accuracy about $100 \mu \mathrm{m}$.

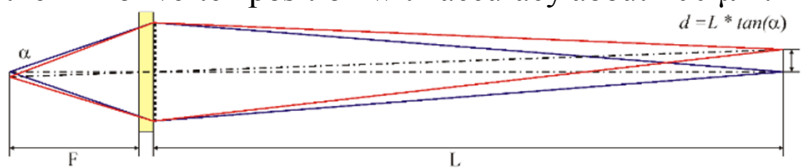

Fig. 5. Shift of the surface vertex marked point due to hologram tilt with respect to interferometer.

\section{References}

1. A.G. Poleshchuk, V. P Korolkov, R. K. Nasyrov, J.M. Asfour, Proc SPIE 7102, 710206 (2008)

2. A.G Poleshchuk, Proc. SPIE 7544, 75443L-1 (2010)

3. A.G. Poleshchuk, Proc. SPIE 7133, 713333-1 (2009)

4. A.P. Semenov, M.A. Abdulkadyrov, V.E Patrikeev, Proc. SPIE 9683, 968314-1 (2016)

5. A.P. Semenov, M.A. Abdulkadyrov, V.E. Patrikeev, Proc. SPIE 9912, 991246-1 (2016)

6. M. A. Abdulkadyrov, A. P. Semenov, S. P. Belousov, N. M. Vladimirov, A. N. Ignatov, V. E. Patrikeev, V. V. Pridnya, A. V. Polyanchikov, R. K. Nasyrov, O. Pirnay, C. Flebus, C. Yesilyaprakd, O. Keskine, Proc. SPIE 10706, 1070631-1 (2018) 